\title{
No effect of pure oxygen inhalation on headache induced by glyceryl trinitrate
}

\author{
Dorthe Daugaard • Peer Tfelt-Hansen • \\ Lars Lykke Thomsen · Helle Klingenberg Iversen • \\ Jes Olesen
}

Received: 27 August 2009/ Accepted: 11 January 2010/Published online: 9 February 2010

(C) Springer-Verlag 2010

\begin{abstract}
Inhalation of hyperbaric oxygen has been used as an experimental treatment for migraine and pure oxygen is an established treatment for cluster headache. Intravenous glyceryl trinitrate (GTN) is an established headache model. In the present study the possibility of decreasing the headache by inhalation of pure oxygen was explored in a double-blind crossover design in 18 healthy subjects. Inhalation of air served as placebo. The subjects received intravenous GTN $(0.25 \mu \mathrm{g} / \mathrm{kg} / \mathrm{min})$ for $20 \mathrm{~min}$. Headache was scored for $85 \mathrm{~min}$. Sixteen of $18(89 \%)$ subjects experienced GTN-induced headache after $\mathrm{O}_{2}$-inhalation and 17/18 (94\%) experienced GTN-induced headache after air. The mean peak headache scores were 1.9 and 2.4, respectively, on a numerical scale of $0-10$. Oxygen inhalation did not have effect on GTN-induced headache, most likely because the theoretical decrease in NO levels, due to faster metabolism of NO, is too small to be detected in the GTN headache model.
\end{abstract}

Keywords Oxygen · Glyceryl trinitrate - Nitric oxide · Headache

\section{Introduction}

The intravenous glyceryl trinitate (GTN) headache model is well established [1, 2]. It causes a short-lasting headache in healthy volunteers and in migraine and cluster headache

D. Daugaard · P. Tfelt-Hansen $(\bowtie) \cdot$ L. L. Thomsen ·

H. K. Iversen - J. Olesen

Department of Neurology, Danish Headache Centre,

Glostrup Hospital, University of Copenhagen,

Glostrup, 2600 Copenhagen, Denmark

e-mail: ptha@glo.regionh.dk patients, the so-called immediate GTN headache [1, 2]. In addition, GTN causes a delayed headache in cluster headache [3] and migraine patients [4]. The immediate GTN headache is most likely due to a vasodilator effect of GTN on the extracerebral circulation [5] whereas the delayed headache/migraine is elicited by other mechanisms.

Administration of $\mathrm{N}$-acetylcysteine, a sulfhydryl group donor, enhanced the headache-inducing effect of a GTN infusion in healthy volunteers [6]. This effect of $N$-acetylcysteine is most likely due to enhanced formation of NO from GTN.

In a recent Cochrane review [7] it was concluded that there was some evidence that hyperbaric oxygen was effective for the termination of acute migraine, and weak evidence that normobaric oxygen was similarly effective in cluster headache.

In the present study we tried to decrease the levels of NO in our GTN headache model by administering pure oxygen in a dose, $7 \mathrm{l} / \mathrm{min}$ which has been shown to be effective in the treatment of cluster headache in a doubleblind trial $[8,9]$ and in clinical practice $[8,10]$. Increased oxygen concentration most likely decreases NO concentration by oxidization of $\mathrm{NO}$ to $\mathrm{NO}_{3}$ and $\mathrm{NO}_{2}$.

Healthy volunteers were investigated in a crossover design.

\section{Methods}

Subjects

The study followed a randomized, double-blind, placebocontrolled, and cross-over design. The 2 study days were scheduled at least 7 days apart and included 18 healthy volunteers who had no prior history of migraine and who did not experience tension-type headache more than once a month. Their median age was 23 years (range 
20-33 years), the male:female ratio was $1: 1$, and the median body weight $65 \mathrm{~kg}$ (range $52-86 \mathrm{~kg}$ ). Prior to entry the subjects were challenged with $0.25 \mathrm{mg}$ of GTN sublingually and were included only if they experienced headache. The participants gave informed consent prior to inclusion, and it was emphasized that they were free to withdraw at any time. The study was approved by The Ethics Committee of Copenhagen County.

\section{Procedure}

No caffeine containing beverages, alcohol, tobacco, or medication were allowed for the preceding $12 \mathrm{~h}$ prior to the study, and all had to be headache-free at start. After 30 min of rest, either pure oxygen or placebo (atmospheric air), $7 \mathrm{l} / \mathrm{min}$, was inhaled for $35 \mathrm{~min}$ through a line fastened to an inflatable anesthesia mask. The gas bottles were hidden to the investigator and the participants. The proper gas was connected to the mask by a laboratory technician. GTN, $0.25 \mu \mathrm{g} / \mathrm{kg} / \mathrm{min}$, was infused for 20 min through a i.v. line in the right cubital vein using a volume-directed infusion pump (Braun Perfusor, Secura). Headache was rated on a verbal 0-10 numerical scale; 1 representing pre-pain, 5 a moderate headache, and 10 the worst possible headache. At pain scores above 0 the headache and accompanying symptoms were characterized according to a standardized questionnaire covering the IHS criteria for migraine without aura (IHS-classification, 12). The study ended $85 \mathrm{~min}$ after the start of the oxygen/placebo inhalation.

\section{Statistics}

Statistical analyses were performed with Statgraphic 7.0. Randomization and Wilcoxon-Pratt statistics were performed using Medstat, $p<0.05$ was chosen as the statistical level of significance. Differences in the total, summed, and peak headache scores between the two study days were analyzed using Wilcoxon-Pratt's test.

\section{Results}

All subjects completed the study. No serious adverse events were seen during or after the infusion of GTN.

Sixteen of 18 (89\%) subjects experienced headache after the $\mathrm{O}_{2}$ inhalation and $17 / 18$ (94\%) on the day with air. The mean peak headache score in was $1.9\left(\mathrm{O}_{2}\right)$ and 2.4 (air).

Oxygen did not significantly alter the summed headache scores or the peak headache scores (Wilcoxon-Pratt; summed $p=0.11$, peak $p=0.25$; Fig. 1 ).

The headache showed similar characteristics in the majority of the subjects. It was mild, bilateral, pressing, mainly fronto-temporal headache of short duration without

Fig. 1 Individual headache scores in the laboratory, 0-85 min after glyceryl trinitrate (with means in bold), $n=18$. There was no difference between oxygen and air inhalation1
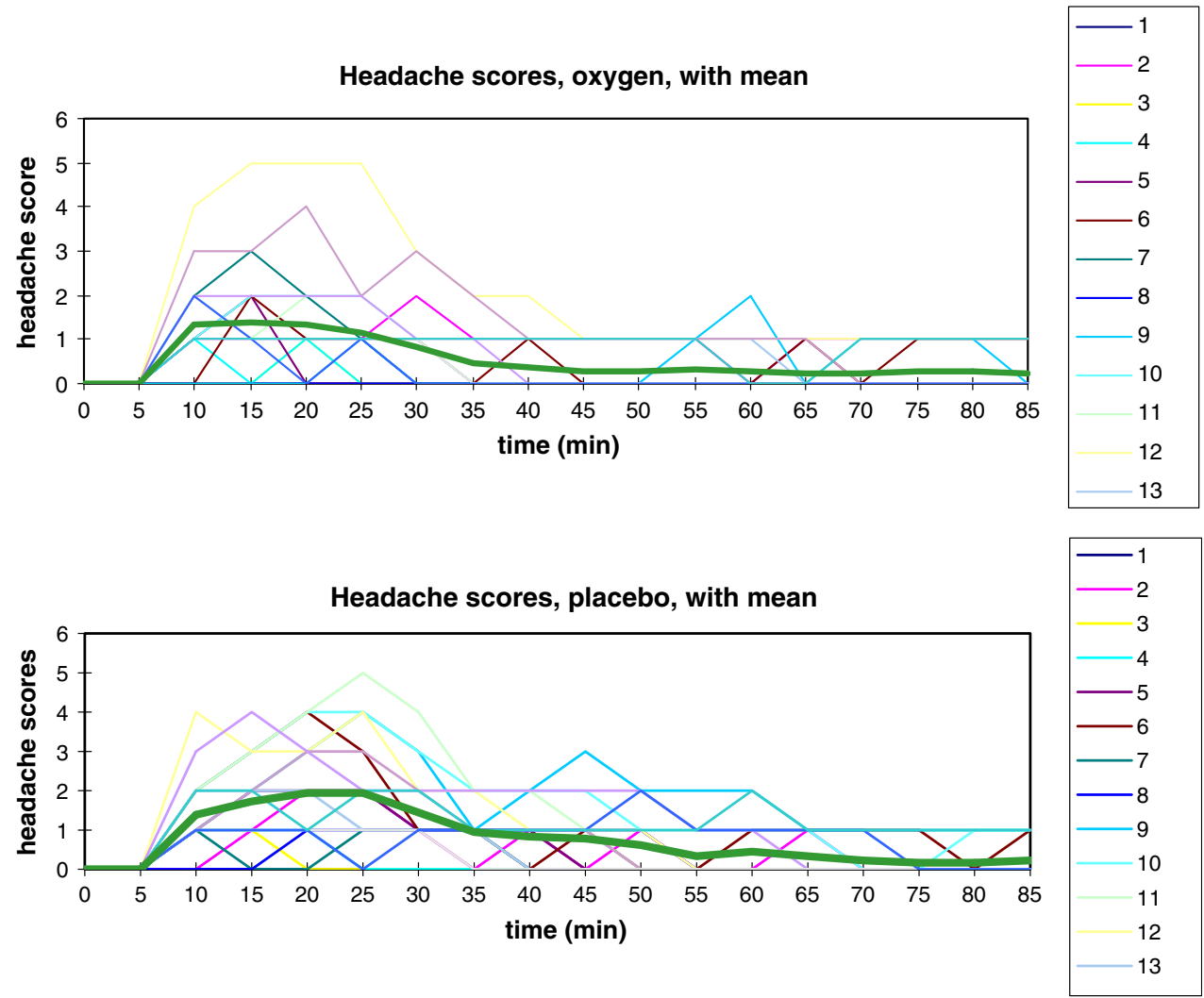
accompanying symptoms. No subject fulfilled the diagnostic criteria for migraine without aura [11].

\section{Discussion}

The rationale for this study was the fact that increased oxygen tension will tend to increase the metabolism of NO. In an aqueous solution NO reacts rapidly and spontaneously with oxygen to produce mainly nitrite via the intermediary formation of nitrogen dioxide and dinitrogen trioxide [12].

A reduction in $\mathrm{pO}_{2}$ causes an increase in $\mathrm{NO}$ in some $[13,14]$ but not all $[15,16]$ studies. Theoretically, hypoxia could cause an increase in the effect of glyceryl trinitrate (GTN). This effect cannot be explored experimentally in humans outside a hypobaric chamber. In contrast, an increase in $\mathrm{pO}_{2}$ causes a shortening of the half-life of $\mathrm{NO}$ [17] due to increased metabolism and most likely a decrease in the effect of GTN. This possible effect of pure oxygen was explored in the present study.

The major result of the present study was that a therapeutic dose of oxygen inhalation (7 1/min) [8], did not reduce the headache induced by GTN infusion $(0.25 \mu \mathrm{g} / \mathrm{kg} / \mathrm{min})$ in healthy volunteers.

Theoretically, this "negative" result could be due either to the fact that the GTN headache model is not sensitive enough to detect changes in NO levels or to a lack of oxygen to counteract the effect of GTN/NO.

That the GTN model is sensitive to changes in NO is demonstrated by the fact that a dose-response curve for the headache effect of GTN could be constructed [2] and by the fact that the sulfhydryl donor, $N$-acetylcysteine, increased the NTG-induced headache [6].

The dose of oxygen by inhalation was $71 / \mathrm{min}$ [8] but there was no effect on NTG-induced headache, most likely because the obtained increase of $\mathrm{pO}_{2}$ was not large enough to counteract the effect of GTN. Thus, in isolated guinea pig hearts increasing oxygen tension in aqueous solutions from 150 to $700 \mathrm{mmHg}$ decreased NO half-life (5.2 s) by $32 \%$ [17]. Hundred percent oxygen inhalation in normal adults increases $\mathrm{pO}_{2}$ to about $600 \mathrm{mmHg}$ [18]. Based on the in vitro in guinea pig study [17] this $\mathrm{pO}_{2}(600 \mathrm{mmHg})$ in humans may result in too small a decrease in NO levels to be detected in our headache model.

Our results suggest that the effect of pure oxygen in cluster headache may not be mediated via a reduction of NO.

Acknowledgments The study was supported by The Migraine Trust, UK, by the Danish Headache Society, and by the Lundbeck Foundation via the Lundbeck Foundation Center for Neurovascular Signaling (LUCENS), Denmark. Statistical advice was provided by statistician Lene Theil Skovgaard, Department of Biostatistics,
University of Copenhagen, Denmark. We are grateful for the assistance of laboratory technicians Bente Leisner and Kirsten Enghave.

Conflict of interest None.

\section{References}

1. Kruuse C, Afridi SK (2006) Human models of the headaches. In: Olesen J, Goadsby PJ, Ramadan NM, Tfelt-Hansen P, Welch KMA (eds) The headaches, 3rd edn. Lippincott Williams \& Wilkins, Philadelphia, pp 221-229

2. Iversen HK, Olesen J, Tfelt-Hansen P (1989) Intravenous nitroglycerin as an experimental model of vascular headache. Basic characteristics. Pain 38:17-24

3. Ekbom K (1968) Nitroglycerin as a provocative agent in cluster headache. Arch Neurol 19:487-493

4. Thomsen LL, Kruuse C, Iversen HK, Olesen J (1994) A nitric oxide donor (nitroglycerin) triggers genuine migraine attacks. Eur J Neurol 1:73-80

5. Christiansen I, Iversen HK, Olesen J, Tfelt-Hansen P (2008) Nitric oxide-induced headache may arise from extracerebral arteries as judged from tolerance to isosorbide 5-mononitrate. J Headache Pain 9:215-220

6. Iversen HK (1992) $N$-acetylcysteine enhances nitroglycerininduced headache and cranial arterial responses. Clin Pharmacol Ther 52:125-133

7. Bennett MH, French C, Schnabel A, Wasiak J, Kranke P (2008) Normobaric and hyperbaric oxygen therapy for migraine and cluster headache. Cochrane Database Syst Rev 3:CD005219

8. Sandrini G, Ward TN (2006) Acute treatment of cluster headache. In: Olesen J, Goadsby PJ, Ramadan NM, Tfelt-Hansen P, Welch KMA (eds) The headaches, 3rd edn. Lippincott Williams \& Wilkins, Philadelphia, pp 803-807

9. Fogan L (1985) Treatment of cluster headache. A double-blind comparison of oxygen v air inhalation. Arch Neurol 42:362-363

10. Kudrow L (1981) Response of cluster headache attacks to oxygen inhalation. Headache 21:1-4

11. Headache Classification Committee of the International Headache Society (2004) Classification and diagnostic criteria for headache disorders, cranial neuralgia and facial pain, 2 nd edn. Cephalalgia 24(Suppl 1):1-164

12. Purwanti E, Mukhopadhyay B (2009) Conversion of $\mathrm{NO}_{2}$ to $\mathrm{NO}$ by reduced coenzyme $\mathrm{F}_{420}$ protects mycobacteria from nitrosactive damage. PNAS 106:6333-6338

13. Hampl V, Cornfield DN, Cowan NJ, Archer SL (1995) Hypoxia potentiates nitric oxide synthesis and transiently increases cytosolic calcium levels in pulmonary artery endothelial cells. Eur Respir J 8:515-522

14. Xu XP, Pollock JS, Tanner MA, Myers PR (1995) Hypoxia activates nitric oxide synthase and stimulates nitric oxide production in porcine coronary resistance arteriolar endothelial cells. Cardiovasc Res 30:841-847

15. Shaul PW, Wells LB, Horning KM (1993) Acute and prolonged hypoxia attenuate endothelial nitric oxide production in rat pulmonary arteries by different mechanisms. J Cardiovascul Pharmacol 22:819-827

16. Whorton AR, Simonds DB, Piantadosi CA (1997) Regulation of nitric oxide synthesis by oxygen in vascular endothelial cells. Am J Physiol 272:L1161-L1166

17. Kelm M, Schrader J (1990) Control of coronary vascular tone by nitric oxide. Circ Res 66:1561-1575

18. Koeff ST, Tsao MU, Vadnay A, Wilson TO, Wilson JL (1962) Continuous measurements of intravascular oxygen tension in normal adults. J Clin Invest 41:1125-1133 